\title{
ADDENDUM TO THE ARTICLE "ON THE DIRICHLET TO NEUMANN PROBLEM FOR THE 1-DIMENSIONAL CUBIC NLS EQUATION ON THE HALF-LINE"
}

\author{
D. C. ANTONOPOULOU ${ }^{\$}$ AND S. KAMVISSIS*
}

\begin{abstract}
We present a short note on the extension of the results of [1] to the case of non-zero initial data. More specifically, the defocusing cubic NLS equation is considered on the half-line with decaying (in time) Dirichlet data and sufficiently smooth and decaying (in space) initial data. We prove that for this case also, and for a large class of decaying Dirichlet data, the Neumann data are sufficiently decaying so that the Fokas unified method for the solution of defocusing NLS is applicable.
\end{abstract}

\section{INTRODUCTION}

In this note, we consider the defocusing non-linear Schrödinger equation (NLS) with cubic nonlinearity, posed on the real positive semi-axis $\mathbb{R}^{+}$

$$
\mathrm{i} q_{t}+q_{x x}-2|q|^{2} q=0, \quad x>0, \quad 0<t<+\infty,
$$

and initial-boundary data

$$
\begin{aligned}
& q(x, 0)=q_{0}(x), \quad 0 \leq x<+\infty \\
& q(0, t)=Q(t), \quad 0 \leq t<+\infty
\end{aligned}
$$

where $q_{0}, Q$ are classical functions satisfying the compatibility condition $q_{0}(0)=Q(0)$.

Existence and uniqueness of solution for the problem (1.1)-(1.2) with $q_{0} \in H^{2}, Q \in C^{2}$ and $q_{0}(0)=Q(0)$, have been established in [2].

Our aim here is to show that under specific conditions on $q_{0}$ and $Q$, the function $q_{x}(0, t)$ is in $L^{1}(d t)$ and hence the initial/boundary value problem admits a full analysis via the unified inverse scattering method initiated and studied by Fokas and collaborators.

In a previous publication [1] we considered the simplified problem with zero initial data. It turns out that the result can be easily extended to the case of general initial data satisfying particular decay and smoothness conditions. For the convenience of the reader we provide here a self-contained proof with full details.

\section{An $L^{2}(0, \infty)$ estimate for the Neumann data}

We begin with some notations: $(\cdot, \cdot)$ will denote the $L^{2}(0, \infty)$ inner product in space variables, and $\|\cdot\|$ the induced norm. The symbol $\|\cdot\|_{4}$ is used for the $L^{4}(0, \infty)$ norm in space, while for any

* Department of Pure and Applied Mathematics, University of Crete, GR-700 13 Heraklion, Greece, and, Institute of Applied and Computational Mathematics, FORTH, GR-711 10 Heraklion, Greece, email: spyros@tem.uoc.gr.

\$ Department of Mathematics, University of Chester, Thornton Science Park, CH2 4NU, UK, and, Institute of Applied and Computational Mathematics, FORTH, GR-711 10 Heraklion, Greece, email: d.antonopoulou@chester.ac.uk. 
integer $p \geq 1,\|\cdot\|_{L^{p}(0, t)}$ will denote the $L^{p}$ norm in the time interval $(0, t)$. The symbol $c$ will be used to denote a generic positive constant.

We emphasize here the following decay condition for the solution $q$ of the NLS problem (1.1)-(1.2)

$$
q(x, t) \rightarrow 0 \text { as } \quad x \rightarrow \infty \text { for any } t \geq 0,
$$

which is crucial for our arguments, and is of course provided by the result in [2].

Theorem 2.1. Let $q$ be the unique global classical solution $q \in C^{1}\left(L^{2}\right) \cap C^{0}\left(H^{2}\right)$ of the problem (1.1) -(1.2), with $Q \in C^{2}$ and $Q(0)=q_{0}(0)$, under the decay assumption (2.1). It holds that for any $t>0$

$$
\begin{aligned}
\left(\int_{0}^{t}\left|q_{x}(0, s)\right|^{2} d s\right)^{1 / 2}= & \left\|q_{x}(0, \cdot)\right\|_{L^{2}(0, t)} \\
\leq & c\|q(0, \cdot)\|_{L^{2}(0, t)}^{2}+c\left\|q_{t}(0, \cdot)\right\|_{L^{2}(0, t)}^{2}+\|q(0, \cdot)\|_{L^{4}(0, t)}^{4} \\
& +c\left\|q_{0}\right\|^{2}+c\left\|q_{0 x}\right\|^{2}+c\left\|q_{0}\right\|_{L^{4}(0, \infty)}^{4}
\end{aligned}
$$

where $c$ is a positive constant. The previous estimate is true also when $t$ is replaced by $\infty$.

Proof. Multiplying (1.1) by $\bar{q}$ and integrating in $x \in(0, \infty)$ we obtain after applying integration by parts

$$
\mathrm{i}\left(q_{t}, q\right)-\left\|q_{x}\right\|^{2}-q_{x}(0, t) \bar{q}(0, t)-2\left(|q|^{2} q, q\right)=0 .
$$

Taking imaginary parts we arrive at

$$
\frac{d}{d t}\|q\|^{2}=2 \operatorname{Im}\left\{q_{x}(0, t) \bar{q}(0, t)\right\}
$$

which after integration in time gives

$$
\|q(\cdot, t)\|^{2} \leq\|q(\cdot, 0)\|^{2}+2\left\|q_{x}(0, \cdot)\right\|_{L^{2}(0, t)}\|q(0, \cdot)\|_{L^{2}(0, t)} .
$$

Multiplying now (1.1) by $\bar{q}_{t}$ and integrating in $x \in(0, \infty)$ we obtain

$$
\mathrm{i}\left\|q_{t}\right\|^{2}-\left(q_{x}, q_{x t}\right)-q_{x}(0, t) \bar{q}_{t}(0, t)-2\left(|q|^{2} q, q_{t}\right)=0,
$$

while taking real parts and since

$$
\frac{d}{d t}\left[|q|^{2}|q|^{2}\right]=4 \operatorname{Re}\left\{|q|^{2} q \bar{q}_{t}\right\}
$$

we have

$$
\frac{d}{d t}\left\|q_{x}\right\|^{2}=-\frac{d}{d t}\left\||q|^{2}\right\|^{2}-2 \operatorname{Re}\left\{q_{x}(0, t) \bar{q}_{t}(0, t)\right\} .
$$

Integration in time yields

$$
\left\|q_{x}(\cdot, t)\right\|^{2}-\left\|q_{x}(\cdot, 0)\right\|^{2}=-\left\||q(\cdot, t)|^{2}\right\|^{2}+\left\||q(\cdot, 0)|^{2}\right\|^{2}-2 \int_{0}^{t} \operatorname{Re}\left\{q_{x}(0, s) \bar{q}_{t}(0, s)\right\} d s,
$$

and thus,

$$
\left\|q_{x}(\cdot, t)\right\|^{2}+\|q(\cdot, t)\|_{L^{4}(0, \infty)}^{4} \leq\left\|q_{x}(\cdot, 0)\right\|^{2}+\|q(\cdot, 0)\|_{L^{4}(0, \infty)}^{4}+2\left\|q_{x}(0, \cdot)\right\|_{L^{2}(0, t)}\left\|q_{t}(0, \cdot)\right\|_{L^{2}(0, t)} .
$$

We now multiply (1.1) with $\overline{q_{x}}$ integrate in space and take real parts which yields

$$
-\operatorname{Im}\left(q_{t}, q_{x}\right)+\operatorname{Re}\left(q_{x x}, q_{x}\right)-2 \operatorname{Re}\left(|q|^{2} q, q_{x}\right)=0 .
$$


Using that

and the relations

$$
\operatorname{Im}\left(q_{t}, q_{x}\right)=-\frac{1}{2} \mathrm{i} \frac{d}{d t}\left(q, q_{x}\right)-\frac{1}{2} \mathrm{i} q(0, t) \bar{q}_{t}(0, t)
$$

$$
\operatorname{Re}\left(q_{x x}, q_{x}\right)=-\frac{1}{2}\left|q_{x}(0, t)\right|^{2}, \quad \operatorname{Re}\left(|q|^{2} q, q_{x}\right)=-\frac{1}{4}|q(0, t)|^{4},
$$

we obtain

$$
\left|q_{x}(0, t)\right|^{2}=\mathrm{i} \frac{d}{d t}\left(q, q_{x}\right)+\mathrm{i} q(0, t) \bar{q}_{t}(0, t)+|q(0, t)|^{4} .
$$

Integrating the above in time, we get

$$
\int_{0}^{t}\left|q_{x}(0, s)\right|^{2} d s=\mathrm{i}\left(q(\cdot, t), q_{x}(\cdot, t)\right)-\mathrm{i}\left(q(\cdot, 0), q_{x}(\cdot, 0)\right)+\mathrm{i} \int_{0}^{t} q(0, s) \bar{q}_{t}(0, s) d s+\int_{0}^{t}|q(0, s)|^{4} d s,
$$

and so,

$$
\begin{aligned}
\left\|q_{x}(0, \cdot)\right\|_{L^{2}(0, t)}^{2} \leq & \|q(\cdot, t)\|\left\|q_{x}(\cdot, t)\right\|+\|q(\cdot, 0)\|\left\|q_{x}(\cdot, 0)\right\| \\
& +\|q(0, \cdot)\|_{L^{2}(0, t)}\left\|q_{t}(0, \cdot)\right\|_{L^{2}(0, t)}+\|q(0, \cdot)\|_{L^{4}(0, t)}^{4} \\
\leq & c\|q(\cdot, t)\|^{2}+c\left\|q_{x}(\cdot, t)\right\|^{2}+\|q(\cdot, 0)\|\left\|q_{x}(\cdot, 0)\right\| \\
& +\|q(0, \cdot)\|_{L^{2}(0, t)}\left\|q_{t}(0, \cdot)\right\|_{L^{2}(0, t)}+\|q(0, \cdot)\|_{L^{4}(0, t)}^{4}
\end{aligned}
$$

Using now (2.4) and (2.6) we get

$$
\begin{aligned}
\left\|q_{x}(0, \cdot)\right\|_{L^{2}(0, t) \leq}^{2} \leq & \|q(\cdot, 0)\|^{2}+2 c\left\|q_{x}(0, \cdot)\right\|_{L^{2}(0, t)}\|q(0, \cdot)\|_{L^{2}(0, t)} \\
& +c\left\|q_{x}(\cdot, 0)\right\|^{2}+c\|q(\cdot, 0)\|_{L^{4}(0, \infty)}^{4}+2 c\left\|q_{x}(0, \cdot)\right\|_{L^{2}(0, t)}\left\|q_{t}(0, \cdot)\right\|_{L^{2}(0, t)} \\
& +\|q(\cdot, 0)\|\left\|q_{x}(\cdot, 0)\right\|+\|q(0, \cdot)\|_{L^{2}(0, t)}\left\|q_{t}(0, \cdot)\right\|_{L^{2}(0, t)}+\|q(0, \cdot)\|_{L^{4}(0, t)}^{4} \\
\leq & c_{0}\left\|q_{x}(0, \cdot)\right\|_{L^{2}(0, t)}^{2}+c\|q(0, \cdot)\|_{L^{2}(0, t)}^{2}+c\left\|q_{t}(0, \cdot)\right\|_{L^{2}(0, t)}^{2} \\
& +c\|q(\cdot, 0)\|^{2}+c\left\|q_{x}(\cdot, 0)\right\|^{2}+c\|q(\cdot, 0)\|_{L^{4}(0, \infty)}^{4} \\
& +\|q(\cdot, 0)\|\left\|q_{x}(\cdot, 0)\right\|+\|q(0, \cdot)\|_{L^{2}(0, t)}\left\|q_{t}(0, \cdot)\right\|_{L^{2}(0, t)}+\|q(0, \cdot)\|_{L^{4}(0, t)}^{4},
\end{aligned}
$$

where $c_{0}$ can be made as small as we want. Thus, we arrive at the result (2.2), i.e.

$$
\begin{aligned}
\left\|q_{x}(0, \cdot)\right\|_{L^{2}(0, t)}^{2} \leq & c\|q(0, \cdot)\|_{L^{2}(0, t)}^{2}+c\left\|q_{t}(0, \cdot)\right\|_{L^{2}(0, t)}^{2}+\|q(0, \cdot)\|_{L^{4}(0, t)}^{4} \\
& +c\|q(\cdot, 0)\|^{2}+c\left\|q_{x}(\cdot, 0)\right\|^{2}+c\|q(\cdot, 0)\|_{L^{4}(0, \infty)}^{4} .
\end{aligned}
$$

Remark 2.2. Note that the previous estimate provides a bound for

$$
\left\|q_{x}(0, \cdot)\right\|_{L^{2}(0, \infty)}^{2}
$$

when $q_{0}$ is sufficiently smooth.

More specifically, if $q_{0} \in H^{1}(0, \infty) \cap L^{4}(0, \infty)$ and if $q(0, t), q_{t}(0, t)$, have polynomial decay of order $\mathcal{O}\left(t^{-\alpha}\right), \mathcal{O}\left(t^{-\beta}\right)$ respectively as $t \rightarrow \infty$, with $\alpha>1 / 2$ and $\beta>1 / 2$ then the estimate (2.2) of Theorem 2.1 implies

$$
\int_{0}^{\infty}\left|q_{x}(0, t)\right|^{2} d t<\infty
$$


In addition, the estimate (2.10), together with (2.4) and (2.6) yield that there exists $c>0$ independent of $t$, such that for any $t \geq 0$

$$
\begin{gathered}
\|q(\cdot, t)\| \leq c, \\
\left\|q_{x}(\cdot, t)\right\| \leq c
\end{gathered}
$$

and

$$
\|q(\cdot, t)\|_{L^{4}(0, \infty)} \leq c .
$$

\section{Decay of solution as $t \rightarrow \infty$}

In the sequel, we prove, under certain assumptions for the initial data, that the solution of (1.1)(1.2) decays to 0 for any $x$ as $t \rightarrow \infty$. For this, we first establish that the $L^{4}$ norm of the solution decays like $\mathcal{O}\left(t^{-\frac{1}{4}}\right)$ as $t \rightarrow \infty$.

Theorem 3.1. Let $q$ be the unique global classical solution $q \in C^{1}\left(L^{2}\right) \cap C^{0}\left(H^{2}\right)$ of the problem (1.1) -(1.2), with $Q \in C^{2}$ and $Q(0)=q_{0}(0)$, under the assumptions (2.1).

Assume that $q_{0} \in H^{1}(0, \infty) \cap L^{4}(0, \infty)$ and $x q_{0} \in L^{2}(0, \infty)$. Furthermore, assume that as $t \rightarrow \infty$

$$
q(0, t)=\mathcal{O}\left(t^{-\alpha}\right), \quad q_{t}(0, t)=\mathcal{O}\left(t^{-\beta}\right), \quad \text { for } \alpha>3 / 2 \quad \text { and } \beta>5 / 2 .
$$

It holds that there exists a positive constant $c$ independent of $t$ such that

$$
\int_{0}^{\infty}|q(x, t)|^{4} d x:=\|q(\cdot, t)\|_{4}^{4} \leq \frac{c}{t} \quad \text { for any } t \geq 1 .
$$

Proof. Again let the Dirichlet data be

$$
Q(t):=q(0, t)
$$

and let us denote the Neumann data by

$$
P(t):=q_{x}(0, t)
$$

We set

$$
y(t)=\operatorname{Im} \int_{0}^{\infty} x \bar{q}(x, t) q_{x}(x, t) d x,
$$

to obtain (cf. the analytical proof of the specific identity in [1])

$$
\begin{aligned}
\partial_{t}\left(t^{2} \int_{0}^{\infty}|q|^{4} d x\right)= & \frac{1}{4} \partial_{t}\left[4 t y-\int_{0}^{\infty} x^{2}|q(x, t)|^{2} d x-4 t^{2} \int_{0}^{\infty}\left|q_{x}\right|^{2} d x\right] \\
& -t \operatorname{Re}\{P \bar{Q}\}-2 t^{2} \operatorname{Re}\left\{P \bar{Q}_{t}\right\}+t \int_{0}^{\infty}|q|^{4} d x
\end{aligned}
$$

Integrating the above in time in the interval $(0, t)$, we get

$$
\begin{aligned}
t^{2}\|q(\cdot, t)\|_{4}^{4}= & \frac{1}{4}\left[4 t y-\int_{0}^{\infty} x^{2}|q(x, t)|^{2} d x+\int_{0}^{\infty} x^{2}|q(x, 0)|^{2} d x-4 t^{2} \int_{0}^{\infty}\left|q_{x}(x, t)\right|^{2} d x\right] \\
& -\int_{0}^{t} r \operatorname{Re}\{P(r) \bar{Q}(r)\} d r-2 \int_{0}^{t} r^{2} \operatorname{Re}\left\{P(r) \bar{Q}_{r}(r)\right\} d r+\int_{0}^{t} r\|q(\cdot, r)\|_{4}^{4} d r .
\end{aligned}
$$

Observe now that

$$
4 t y=\int_{0}^{\infty}\left[x^{2}|q|^{2}+4 t^{2}\left|q_{x}\right|^{2}-\left|x q+2 \mathrm{i} t q_{x}\right|^{2}\right] d x
$$


while by (3.2) we have

$$
\begin{aligned}
t^{2}\|q(\cdot, t)\|_{4}^{4}=\frac{1}{4}[ & \int_{0}^{\infty}\left(x^{2}|q(x, t)|^{2}+4 t^{2}\left|q_{x}(x, t)\right|^{2}-\left|x q+2 \mathrm{i} t q_{x}(x, t)\right|^{2}-x^{2}|q(x, t)|^{2}+x^{2}|q(x, 0)|^{2}\right) d x \\
& \left.-4 t^{2} \int_{0}^{\infty}\left|q_{x}(x, t)\right|^{2} d x\right] \\
& -\int_{0}^{t} r \operatorname{Re}\{P(r) \bar{Q}(r)\} d r-2 \int_{0}^{t} r^{2} \operatorname{Re}\left\{P(r) \bar{Q}_{r}(r)\right\} d r+\int_{0}^{t} r\|q(\cdot, r)\|_{4}^{4} d r \\
= & \frac{1}{4}\left[-\int_{0}^{\infty}\left|x q(x, t)+2 \mathrm{i} t q_{x}(x, t)\right|^{2} d x\right]+\frac{1}{4} \int_{0}^{\infty} x^{2}|q(x, 0)|^{2} d x \\
& -\int_{0}^{t} r \operatorname{Re}\{P(r) \bar{Q}(r)\} d r-2 \int_{0}^{t} r^{2} \operatorname{Re}\left\{P(r) \bar{Q}_{r}(r)\right\} d r+\int_{0}^{t} r\|q(\cdot, r)\|_{4}^{4} d r .
\end{aligned}
$$

Hence, (3.4) gives

$$
t^{2}\|q(\cdot, t)\|_{4}^{4} \leq \int_{0}^{t} r\|q(\cdot, r)\|_{4}^{4} d r+\frac{1}{4} \int_{0}^{\infty} x^{2}|q(x, 0)|^{2} d x+F\left(P, Q, Q_{t}, t\right),
$$

for

$$
F\left(P, Q, Q_{t}, t\right):=-\int_{0}^{t} r \operatorname{Re}\{P(r) \bar{Q}(r)\} d r-2 \int_{0}^{t} r^{2} \operatorname{Re}\left\{P(r) \bar{Q}_{r}(r)\right\} d r .
$$

Next, we prove that there exists $c>0$ independent of $t$ such that for any $t \geq 0$,

$$
\left|F\left(P, Q, Q_{t}, t\right)\right| \leq c .
$$

Indeed, if $a>3 / 2$ and $\beta>5 / 2$, then we have for $F=F\left(P(t), Q(t), Q_{t}(t), t\right)$

$$
\begin{aligned}
|F| & \leq c\left(\int_{0}^{t}|P(r)|^{2} d r\right)^{1 / 2}\left(\int_{0}^{t} r^{2}|Q(r)|^{2} d r\right)^{1 / 2}+c\left(\int_{0}^{t}|P(r)|^{2} d r\right)^{1 / 2}\left(\int_{0}^{t} r^{4}\left|Q_{r}(r)\right|^{2} d r\right)^{1 / 2} \\
& \leq c\left(\int_{0}^{t}|P(r)|^{2} d r\right)^{1 / 2} \leq c,
\end{aligned}
$$

since (2.10) is true. Thus, (3.6) follows.

Since

$$
\int_{0}^{\infty} x^{2}|q(x, 0)|^{2} d x<\infty
$$

then using (3.6) at (3.5), we obtain that there exists $c>0$ independent of $t$ such that

$$
t^{2}\|q(\cdot, t)\|_{4}^{4} \leq \int_{0}^{t} r\|q(\cdot, r)\|_{4}^{4} d r+c .
$$

So, since $\int_{0}^{1}\|q(\cdot, t)\|_{4}^{4} d t$ is bounded as (2.13) is true, we obtain (3.1), cf. also the analytical proof of [1].

Remark 3.2. An immediate outcome of the last theorem (as well as its counterpart, Theorem 3.1 in [1]), is the absence of any solitons for the defocusing NLS on the half-line. This result is arrived at without the need of employing the unified theory of Fokas. Of course, it does follow from that theory that there are no solitons for this problem (see [4]) and even more one has a complete rigorous asymptotic description of the decaying behavior by analyzing the Riemann-Hilbert problem asymptotically for large times (see [3]). But one has to remember that the above theorem is essential 
in providing a class of data admissible for the unified theory to begin with. It is thus indispensible in justifying the use of the unified theory, which is a prerequisite for the Riemann-Hilbert formulation.

Now we are ready to prove the next main theorem, which establishes the decay of defocusing NLS solution as $t \rightarrow \infty$, uniformly in space.

Theorem 3.3. Let $q$ be the unique global classical solution $q \in C^{1}\left(L^{2}\right) \cap C^{0}\left(H^{2}\right)$ of the problem (1.1)-(1.2), with $Q \in C^{2}$ and $Q(0)=q_{0}(0)$, under the assumptions (2.1).

Assume that $q_{0} \in H^{1}(0, \infty) \cap L^{4}(0, \infty)$ and $x q_{0} \in L^{2}(0, \infty)$. Furthermore, assume that as $t \rightarrow \infty$

$$
q(0, t)=\mathcal{O}\left(t^{-\alpha}\right), \quad q_{t}(0, t)=\mathcal{O}\left(t^{-\beta}\right), \quad \text { for } \alpha>3 / 2 \quad \text { and } \beta>5 / 2 .
$$

It holds that

$$
\lim _{t \rightarrow \infty} q(x, t)=0
$$

for any $x>0$.

Proof. Indeed, we have by Theorem 3.1

$$
\lim _{t \rightarrow \infty}\|q(\cdot, t)\|_{4} \leq \lim _{t \rightarrow \infty} \frac{c}{t^{1 / 4}}=0 .
$$

Using that

we have

$$
\frac{d}{d x} q^{3}(x, t)=3 q^{2}(x, t) q_{x}(x, t)
$$

$$
\begin{aligned}
\left|q^{3}(x, t)\right| & =\left|q^{3}(0, t)+\int_{0}^{x} 3 q^{2}(y, t) q_{x}(y, t) d y\right| \\
& \leq|q(0, t)|^{3}+c\left(\int_{0}^{\infty}|q(y, t)|^{4} d y\right)^{1 / 2}\left(\int_{0}^{\infty}\left|q_{x}(y, t)\right|^{2} d y\right)^{1 / 2} .
\end{aligned}
$$

So, under the assumption of decaying initial data $q(0, t) \rightarrow 0$ as $t \rightarrow \infty$, and since by Remark 2.2 we have (cf. (2.12) )

we conclude from (3.8) that

$$
\left\|q_{x}(\cdot, t)\right\| \leq c
$$

$$
\lim _{t \rightarrow \infty}\left|q^{3}(x, t)\right| \leq c \lim _{t \rightarrow \infty}\|q(\cdot, t)\|_{4}^{2}=0
$$

\section{An $L^{1}(0, \infty)$ estimate for the Neumann data}

At this Section, we shall use the result of Theorem 3.3, i.e. that for all positive $x$,

$$
\lim _{t \rightarrow \infty} q(x, t)=0,
$$

in order to prove, under the assumptions of Theorem 3.3 of course, an $L^{1}(0, \infty)$ bound for $q_{x}(0, t)$.

Consider relation (2.7)

$$
\left|q_{x}(0, t)\right|^{2}=\mathrm{i} \frac{d}{d t}\left(q, q_{x}\right)+\mathrm{i} q(0, t) \bar{q}_{t}(0, t)+|q(0, t)|^{4} .
$$

and multiply with $t^{p}, p>1$ to obtain

$$
t^{p}\left|q_{x}(0, t)\right|^{2}=\mathrm{i} t^{p} \frac{d}{d t}\left(q, q_{x}\right)+\mathrm{i} t^{p} q(0, t) \bar{q}_{t}(0, t)+t^{p}|q(0, t)|^{4} .
$$


Integration of (4.1) in time gives

$$
\begin{aligned}
\int_{0}^{\infty} t^{p}\left|q_{x}(0, t)\right|^{2} d t= & \mathrm{i}\left[t^{p}\left(q(\cdot, t), q_{x}(\cdot, t)\right)\right]_{0}^{\infty}-\mathrm{i} \int_{0}^{\infty} p t^{p-1}\left(q(\cdot, t), q_{x}(\cdot, t)\right) d t \\
& +\mathrm{i} \int_{0}^{\infty} t^{p} q(0, t) \bar{q}_{t}(0, t) d t+\int_{0}^{\infty} t^{p}|q(0, t)|^{4} d t \\
\leq & \lim _{t \rightarrow \infty}\left(t^{p}\|q(\cdot, t)\|\left\|q_{x}(\cdot, t)\right\|\right)+c \int_{0}^{\infty} t^{p-1}\|q(\cdot, t)\|\left\|q_{x}(\cdot, t)\right\| d t \\
& +c \int_{0}^{\infty} t^{p}\left|q(0, t) \| q_{t}(0, t)\right| d t+\int_{0}^{\infty} t^{p}|q(0, t)|^{4} d t .
\end{aligned}
$$

As before, integrating (2.3) in $(t, \infty)$ and using that $\|q(\cdot, \infty)\|=0$, we get

$$
\|q(\cdot, t)\|^{2}=-2 \int_{t}^{\infty} \operatorname{Im}\left\{q_{x}(0, r) \bar{q}(0, r)\right\} d r
$$

which, under the assumptions of Theorem 3.1. by (2.10) yields for any $t \geq 0$

$$
\|q(\cdot, t)\|^{2} \leq c\left(\int_{t}^{\infty}|q(0, r)|^{2} d r\right)^{1 / 2}
$$

Thus, we obtain by (4.2)

$$
\begin{aligned}
\int_{0}^{\infty} t^{p}\left|q_{x}(0, t)\right|^{2} d t \leq & c \lim _{t \rightarrow \infty}\left(t^{p}\left(\int_{t}^{\infty}|q(0, r)|^{2} d r\right)^{1 / 4}\right) \\
& +c \int_{0}^{\infty} t^{p-1}\left(\int_{t}^{\infty}|q(0, r)|^{2} d r\right)^{1 / 4} d t \\
& +c \int_{0}^{\infty} t^{p}|q(0, t)|\left|q_{t}(0, t)\right| d t+\int_{0}^{\infty} t^{p}|q(0, t)|^{4} d t .
\end{aligned}
$$

Furthermore, since $\left\|q_{x}(\cdot, t)\right\|$ is bounded

$$
t^{p}\|q(\cdot, t)\|\left\|q_{x}(\cdot, t)\right\| \leq c t^{p}\left(\int_{t}^{\infty}|q(0, r)|^{2} d r\right)^{1 / 4} \rightarrow 0
$$

if $q(0, t)$ is assured to have a sufficiently fast (polynomial) decay as $t \rightarrow \infty\left(\mathcal{O}\left(t^{-\alpha}\right)\right.$ with $\alpha$ to be specified). Also, we have

$$
\int_{0}^{\infty} t^{p-1}\|q(\cdot, t)\|\left\|q_{x}(\cdot, t)\right\| d t \leq c \int_{0}^{\infty} t^{p-1}\left(\int_{t}^{\infty}|q(0, r)|^{2} d r\right)^{1 / 4} d t \leq c,
$$

again if $q(0, t)$ has a sufficiently fast decay as $t \rightarrow \infty$. The same argument of sufficiently fast decay for $q_{t}(0, t)$ as $t \rightarrow \infty$, (like $\mathcal{O}\left(t^{-\beta}\right)$ with $\beta$ to be specified), together with the previous one, finally gives, using (4.3)

$$
\int_{0}^{\infty} t^{p}\left|q_{x}(0, t)\right|^{2} d t \leq c
$$

if $p>1, \alpha>3 / 2$ and $\beta>5 / 2$, satisfying also

$$
\alpha>2 p+1 / 2, \quad \alpha+\beta>p+1, \quad \alpha>(p+1) / 4 .
$$

Now, we are ready to derive the $L^{1}(0, \infty)$ estimate for $\left|q_{x}(0, t)\right|$. We have the following main theorem. 
Theorem 4.1. Let $q$ be the unique global classical solution $q \in C^{1}\left(L^{2}\right) \cap C^{0}\left(H^{2}\right)$ of the problem (1.1)-(1.2), with $Q \in C^{2}$ and $Q(0)=q_{0}(0)$, under the assumptions (2.1).

Assume that $q_{0} \in H^{1}(0, \infty) \cap L^{4}(0, \infty)$ and $x q_{0} \in L^{2}(0, \infty)$.

If $q(0, t), q_{t}(0, t)$ have a sufficiently fast decay as $t \rightarrow \infty$, that is $\mathcal{O}\left(t^{-\alpha}\right)$ and $\mathcal{O}\left(t^{-\beta}\right)$, for $\alpha>5 / 2$ and $\beta>5 / 2$ respectively, then

$$
\int_{0}^{\infty}\left|q_{x}(0, t)\right| d t<\infty
$$

Proof.

$$
\begin{aligned}
\int_{0}^{\infty}\left|q_{x}(0, t)\right| d t & =\int_{0}^{1}\left|q_{x}(0, t)\right| d t+\int_{1}^{\infty} t^{-\frac{1}{2}-\frac{\varepsilon}{2}} t^{\frac{1}{2}+\frac{\varepsilon}{2}}\left|q_{x}(0, t)\right| d t \\
& \leq c\left(\int_{0}^{1} 1^{2} d t\right)^{1 / 2}\left(\int_{0}^{1}\left|q_{x}(0, t)\right|^{2} d t\right)^{1 / 2}+\left(\int_{1}^{\infty} t^{-1-\varepsilon} d t\right)^{1 / 2}\left(\int_{1}^{\infty} t^{1+\varepsilon}\left|q_{x}(0, t)\right|^{2} d t\right)^{1 / 2} \\
& \leq c+c\left(\int_{0}^{\infty} t^{1+\varepsilon}\left|q_{x}(0, t)\right|^{2} d t\right)^{1 / 2} \leq c
\end{aligned}
$$

where we used (4.4) for $p:=1+\varepsilon$ with $\varepsilon>0$ as small. Note that here, indeed $\alpha>5 / 2>3 / 2$ and $\beta>5 / 2$ and satisfy also

$$
\alpha>2 p+1 / 2, \quad \alpha+\beta>p+1, \quad \alpha>(p+1) / 4 .
$$

\section{ACKNowledgement}

Research funded by ARISTEIA II grant no. 3964 from the General Secretariat of Research and Technology, Greece.

\section{REFERENCES}

[1] D.C. Antonopoulou, S. Kamvissis, On the Dirichlet to Neumann problem for the 1-dimensional Cubic NLS Equation on the Half-Line, Nonlinearity, 28 (2015), pp. 3073-3099.

[2] R. Carrol, Q. Bu, Solution of the forced Nonlinear Schrödinger (NLS) equation using PDE techniques, Applic. Anal., 41 (1991), pp. 33-51.

[3] A. S. Fokas, A. R. Its And L.-Y. Sung, The nonlinear Schrödinger equation on the half-line, Nonlinearity, 18 (2005), pp. 1771-1822.

[4] Jonatan Lenells, Absence of solitons for the defocusing NLS equation on the half-line, arXiv:1412.3495. 